\title{
Why Your Friends Are More Important and Special Than You Think
}

\author{
Thomas U. Grund
}

Stockholm University

\begin{abstract}
A large amount of research finds associations between individuals' attributes and the position of individuals in network structures. In this article, I illustrate how such associations systematically affect the assessment of attributes through network neighbors. The friendship paradox-a general regularity in network contexts, which states that your friends are likely to have more friends than you-becomes relevant and extends to individuals' attributes as well. First, I show that your friends are likely to be better informed (closeness), better intermediaries (betweenness) and more powerful (eigenvector) than you. Second, I suggest more generally that your friends are likely to be more special in their attributes than the population at large. Finally, I investigate the implications of this phenomenon in a dynamic setting. Applying calibrated agent-based simulations, I use a model of attribute adoption to emphasize how structurally introduced experiences penetrate the trajectory of social processes. Existing research does not yet adequately acknowledge this phenomenon.
\end{abstract}

Keywords: social networks; centrality; network neighbors; friendship paradox; agent-based simulations; analytical sociology

Editor(s): Jesper Sørensen, Ezra Zuckerman; Received: December 14, 2013; Accepted: December 27, 2013; Published: April 14, 2014

Citation: Thomas U. Grund. 2014. "Why Your Friends Are More Important and Special Than You Think." Sociological Science 1: 128-140. DOI: 10.15195/v1.a10

Copyright: (C) 2014 Grund. This open-access article has been published and distributed under a Creative Commons Attribution License, which allows unrestricted use, distribution and reproduction, in any form, as long as the original author and source have been credited.

A large number of studies find associations beA tween the positions of individuals in social networks (i.e., centrality) and their attributes, such as tendency to innovate (Tsai 2001), job search success (Granovetter 1973), performance (Brass et al. 2004), or even good looks (Mulford et al. 1998; Tong et al. 2008). In general, such associations are either the result of central individuals adopting certain attributes (such as opinions), or alternatively, of network ties evolving around individuals with certain attributes. An important and overlooked aspect, however, is the way in which such associations affect the experienced distribution of attributes among network neighbors.

Social scientists have long been aware that social structure by itself limits what individuals observe and that it also affects individuals' views of the world. Several decades ago, Feld and Grofman (1977) introduced the class size paradoxalso known in the literature as the friendship paradox. In its simplest form, this paradox postulates that the perceived size of groups is larger than it actually is. Feld and Grofman used the example of college classes with varying sizes, in which most of the students in the classes experience a mean class size that is greater than the true mean. This is due simply to the fact that more students find themselves in larger classes; hence, more students have the experience of being in a large class than being in a small class. Consequently, if one were to ask students about the size of their classes and calculate the mean, one would derive a systematically inflated estimate. Feld (1991) applied this strikingly simple idea to social network contexts and showed that your friendson average - have more friends than you do. The rationale for this regularity is straightforward: individuals with many friends simply appear more often in dyadic relationships, resulting in a systematic mismatch in the distribution of number of friends and the distribution of friends' number of friends.

The general argument of this article is that when there is an association between an attribute and the network position of individuals - no matter how it came about in the first instancesimple embeddedness in network structure gen- 
erates a condition where not only the number of friends' friends, but also the attributes of friends are no longer random (see also Eom and Jo 2014). Following the logic of the friendship paradox, the fact that individuals with many friends appear more often in dyadic friendship relationships means that when an attribute is correlated with network position, some values of the attribute are systematically overrepresented in the assessment of friends' attributes. As soon as there is a correlation between network position and some actor attribute and not everybody has the same number of friends (both conditions are easily met in most empirical settings), exposure to the attributes of others through networks is systematically affected. The existing literature does not adequately acknowledge this phenomenon.

This article elaborates on this in three different ways. First is a discussion of the relevance of the phenomenon for the assessment of other network features. I show that your friends are likely to be better informed (closeness), better intermediaries (betweenness), and more powerful (eigenvector) than you. Second, I outline how the same logic applies to individuals' attributes as well. It is likely that your friends are more special in their attributes than the population at large. Third, I investigate the implications of the friendship paradox in a dynamic setting. Applying calibrated agent-based simulations, I use a model of attribute adoption to emphasize how structurally introduced experiences penetrate the trajectory of social processes. The study emphasizes a previously ignored aspect of the assessment of individuals' attributes through network neighbors.

\section{Centrality}

One arena where this phenomenon plays out is the importance or centrality of nodes in networks. Social network scholars have spent considerable effort developing indicators of centrality as a way to describe individuals' positions in social networks. Centrality is important because it indicates who occupies critical positions in the network. Furthermore, a large array of attributes, such as popularity, opinion leadership, innovation, performance in work teams, individuals' standing in criminal networks, and many more have been associated with it (Ibarra 1993; Morselli, Giguère, and Petit 2007). Various arguments have been brought forward as to what different centrality measures actually mean, where they make sense, and what concepts they represent (Borgatti and Everett 2006; Freeman 1978; Friedkin 1991; Marsden 2002). Perhaps the most frequently used centrality measures are degree, closeness, betweenness, and eigenvector. The first three were proposed by Freeman (1978), and eigenvector was proposed by Bonacich (Bonacich 1972; 1987).

Degree centrality is the most straightforward and intuitive measure. It simply counts, for each individual, the number of outgoing (out-degree) and incoming (in-degree) relationships or network ties. When a network is undirected (network ties do not distinguish between origin and destination), in- and out-degree are the same and are called simply degree. Because of its relative simplicity, degree centrality comes easily to mind, and it has been the sole focus of study of the friendship paradox so far (Christakis and Fowler 2010; Feld 1991). However, degree centrality is a rather local concept. It does not consider the network structure at large but focuses on the immediate network environment (number of friends). Let $y_{i j}$ be a network, where $y_{i j}=1$ means there is a network tie between node $i$ and node $j$ and $y_{i j}=0$ means there is none. For an undirected network, degree centrality of node node $i$ is defined as $C_{\text {degree }}(i)=\sum_{j} y_{i j}$.

In contrast, closeness centrality considers the distance from one actor to all other actors - no matter how far away they are. An actor's closeness centrality is defined as the inverse of the sum of the number of steps it takes to reach all other actors (in the shortest way) (Freeman 1978). Other definitions focus on random walks: on average, how many random steps from one actor to another would it take to reach a focal node (Noh and Rieger 2004)? In any case, closeness is a measure of the speed with which information can spread to the focal actor and is often associated with how well individuals are informed about what happens elsewhere. Higher scores mean that, on average, it takes fewer steps to reach others in the network from this actor; lower scores require longer paths. Usually, the calculation of closeness scores requires the network 
to be connected and undirected. Formally, it is defined as $C_{\text {closeness }}(i)=\sum_{j}\left[d_{i j}\right]^{-1}$, where $d_{i j}$ is the length of the shortest path from node $i$ to node $j$.

Betweenness centrality measures the extent to which a focal actor lies between other actors (Freeman 1978). It is defined as the number of shortest paths (or geodesics) from all actors to all others that pass through the focal node. A definition based on random walks has been proposed here as well: conducting a random walk through the network, how many times would one pass the focal actor (Newman 2005)? Both closeness and betweenness are far more nuanced than degree. An actor may have few friends but still play an important role in the transmission of information through the network as a whole. Betweenness therefore captures the importance of actors as intermediaries or brokers. An actor with high betweenness centrality can potentially influence the spread of information through the network by facilitating, hindering, or even altering the communication between others (Freeman, 1978; Newman, 2003). It is computed as follows: $C_{\text {betweenness }}(i)=\sum_{s} \sum_{t} \frac{\sigma_{s t}(i)}{\sigma_{s t}}$, where $s$ and $t$ are nodes in the network different from $i, \sigma_{s t}$ denotes the number of shortest paths from $s$ to $t$, and $\sigma_{s t}(i)$ is the number of shortest paths from $s$ to $t$ that go through $i$.

Finally, eigenvector centrality is based on the idea that those individuals whose network neighbors are central ought to be more central than those whose neighbors are not (Bonacich 1972; Bonacich 1987). Mathematically, scores correspond to the values of the first eigenvector of the adjacency matrix that defines the network. In more detail, the centrality of each actor is proportional to the sum of the centralities of those actors to whom he or she is connected. One can think of eigenvector as a recursive version of degree centrality. Not just the individual's degree matters, but the degree of those with whom one is connected with as well (and so on). In diffusion contexts, eigenvector centrality is crucial; it captures the idea of multiplied risk and power. Eigenvector centrality is defined as: $C_{\text {eigenvector }}(i)=v_{i}=\frac{1}{\lambda_{\max }(Y)} \cdot \sum_{j} a_{j i} \cdot v_{j}$, with $v=\left(v_{1}, \ldots, v_{n}\right)^{T}$ referring to an eigenvector for the maximum eigenvalue $\lambda_{\max }(Y)$ of the adjacency matrix $Y$, representing the network.

Each of these measures accounts for the prominence of individuals in social networks, but there are considerable conceptual differences between them. Although the different measures of centrality are often correlated, they do not necessarily have to be (Bolland 1988; Rothenberg et al. 1995; Valente et al. 2008), and the mathematical relationships between them are far from obvious.

So far, the friendship paradox has been exclusively thought of in terms of degree centrality. At the time the paradox was discovered, researchers had just begun to investigate nuances between centrality measures and how they are associated with different aspects of social life. But what about the closeness, betweenness and eigenvector scores of friends? Does the friendship-paradox hold here as well? Are your friends, on average, more important than you?

Figure 1 shows a network of mutual friendships among eight girls at "Marketville" high school. While names are fictitious, the data stem from Coleman's (1961) The Adolescent Society; Feld (1991) also uses this example. In Table 1, the first column indicates the number of friends (degree) an individual has and the second column the average number of friends' friends (mean degree of friends), as provided in Feld (1991:1466). The next columns give scores for closeness, betweenness, and eigenvector centrality. On average, individuals' friends seem not only to have more friends themselves (degree), but are also better informed (closeness), are more likely to be brokers (betweenness), and are also more powerful (eigenvector). (See Table 1, last row).

In order to confirm these findings on a larger scale, I draw on the the Dynamics in Networks (DyNet) study's friendship networks in 14 U.S. schools. Data were collected in middle schools in Oregon and California between fall 2008 and spring 2012. All members of the participating middle schools (usually grades six to eight) were interviewed four times each academic year. Students were asked to fill out a questionnaire on demographic characteristics. Furthermore, they were asked to choose from a list of all participating pupils in their school the ones they spend their free time with. As an example, Figure 2 shows the largest component of the network of "Cascade" middle school, with 243 individuals and 


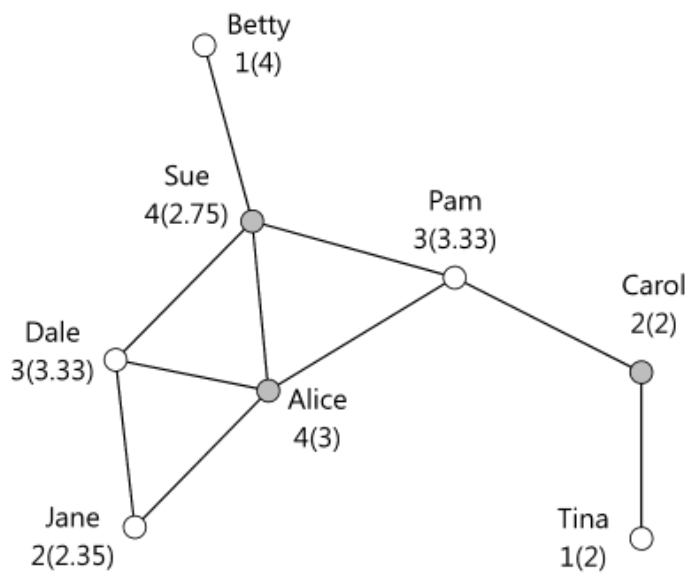

Figure 1: Friendships among eight girls in "Marketville" high school

517 undirected friendship ties. All actors whose degree is smaller than the average degree of their friends are colored in white; those whose degree is larger than the average degree of their friends are colored in grey.

Based on the complete DyNet data, Figure 3 shows results of centrality and friends' centrality calculations. Each school is represented by one data point. The $\mathrm{x}$ axis shows the average centrality score for all students in the school. The y axis shows the average centrality of friends. Both scores are derived the same way as the values listed at the bottom row of Table 1 . The results clearly indicate that, on average, friends have more friends (degree), are better informed (closeness), are more likely to be brokers (betweenness), and are also more powerful (eigenvector) than the average student. All data points are in the upper left triangles of the graphs. Hence, these findings suggest that the friendship paradox is not limited to having more friends but extends to a wide range of other network features as well.

\section{Individual attributes}

More generally, the same logic applies to any individual attribute correlated with degree centrality. In order to illustrate the validity and implications of this intuition, I create simulations using two networks: the real friendship network of "Cascade" middle school as shown in Figure 2 and an Erdõs-Rényi network with exactly the same number of nodes (243) and undirected ties (517). The first network represents a real network, where all sorts of factors played a role in the emergence of ties. The second network is based on realistic assumptions about the number of friendship ties, but structural configurations such as triads are ignored; all ties have the same probability of existing independently of each other (random graph). In both networks I assign all actors an artificial attribute $x$, which is drawn from a normal distribution with a mean of zero and a standard deviation of one. The values of $x$ can be allocated to individuals in such a way that $x$ is correlated with the number of friends (Degree) the individuals have. This is achieved with the corgen command, a built-in function of the $\mathrm{R}$ package ecodist. Other major statistical software packages have similar functions. In the current case, I artificially change the correlation between $x$ and Degree from 0 to 0.9 in steps of 0.1 ; for each configuration I repeat this 100 times. For each combination of network, correlation, and repetition, one can calculate how individuals experience their friends in terms of $x$. Each individual is assigned a score that is the mean of the individual's friends' attribute $x$. These scores are similar to the mean degree of friends' entries in Table 1. I then calculate, for each combination of network, correlation, and repetition, the average score of $x$ for all individuals (grand mean value of $x$ ) and the average score with regards to how all individuals perceive their friends on $x$ (friends mean value of $x$ ). These scores correspond to 
Table 1: Summary of individual's network characteristics and mean network characteristics of friends

\begin{tabular}{|c|c|c|c|c|c|c|c|c|}
\hline & $\begin{array}{l}\mathbb{0} \\
\stackrel{0}{0} \\
\stackrel{0}{0} \\
\stackrel{0}{0}\end{array}$ & 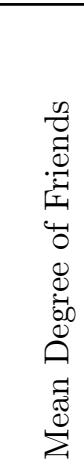 & $\begin{array}{l}\text { D } \\
0 \\
0 \\
0 \\
0 \\
0 \\
0 \\
0\end{array}$ & 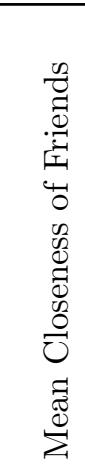 & 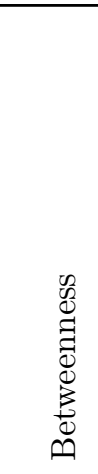 & 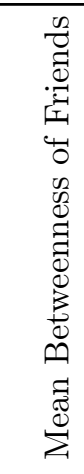 & 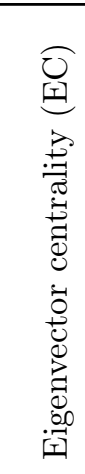 & 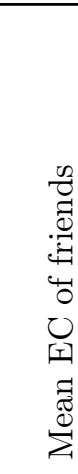 \\
\hline Betty & 1.00 & 4.00 & 0.06 & 0.09 & 0.00 & 7.50 & 0.30 & 0.93 \\
\hline Sue & 4.00 & 2.75 & 0.09 & 0.08 & 7.50 & 4.13 & 0.93 & 0.71 \\
\hline Alice & 4.00 & 3.00 & 0.09 & 0.08 & 5.50 & 4.63 & 1.00 & 0.77 \\
\hline Jane & 2.00 & 3.50 & 0.06 & 0.08 & 0.00 & 3.25 & 0.60 & 0.91 \\
\hline Pam & 3.00 & 3.33 & 0.09 & 0.08 & 10.00 & 6.33 & 0.71 & 0.73 \\
\hline Dale & 3.00 & 3.33 & 0.07 & 0.08 & 1.00 & 4.33 & 0.82 & 0.84 \\
\hline Carol & 2.00 & 2.00 & 0.07 & 0.07 & 6.00 & 5.00 & 0.26 & 0.40 \\
\hline Tina & 1.00 & 2.00 & 0.05 & 0.07 & 0.00 & 6.00 & 0.09 & 0.26 \\
\hline Average & 2.50 & 2.99 & 0.07 & 0.08 & 3.75 & 5.15 & 0.59 & 0.69 \\
\hline
\end{tabular}

the entries in the bottom row of Table 1. Finally, I calculate the difference between these two values and divide it by the standard deviation of this difference over all individuals. In Figure 4, I use boxplots to illustrate the results of the simulation analyses. The $\mathrm{x}$ axis shows the artificially assigned correlation between $x$ and Degree and the $y$ axis shows the standardized deviation between true and perceived average scores of $x$. The data points in the boxplot correspond to the 100 repetitions I used for each combination.

The results are striking. Weak correlationsas low as 0.1 - between $x$ and Degree suffice to generate a significant difference between the true and experienced average scores of $x$. Obviously, as the correlation increases, this difference becomes even more pronounced. Interestingly, despite having the same number of actors and network ties, there is also quite a difference between the real "Cascade" middle school network and the Erdõs-Rényi network with the same density. The effect appears twice as large in the real world network as in the random network. Hence the actual network configuration seems to matter as well. In the empirically calibrated setting, the effect is more pronounced than in the stylized simulation.

So far, I have only focused on static aspects of the friendship paradox. Your friends are likely to have more friends than you do. I have argued that the phenomenon should be viewed more generally and extends to all sorts of individual attributes that are correlated with the number of friends individuals have.

What does that imply? Does the friendship paradox only affect what individuals experience at a specific moment in time or does it also systematically affect the trajectory of social processes, where the behavior or attributes of individuals influence the behavior or attributes of other individuals?

\section{Attribute adoption}

A complex systems point of view, as for example advocated by Thomas Schelling (1971), emphasizes the way in which social phenomena emerge or disappear in light of the interdependencies among the individuals or elements constituting 


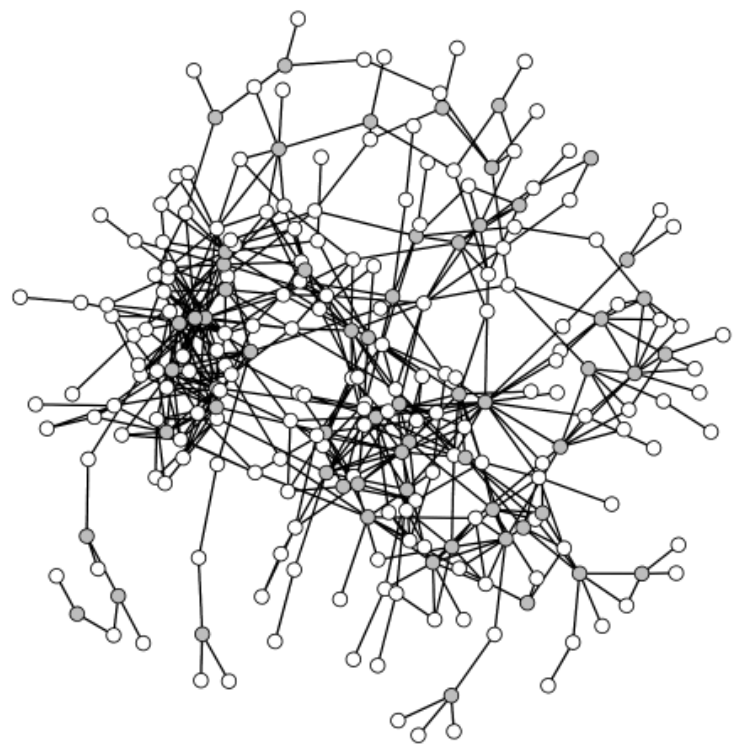

Figure 2: Friendship network of "Cascade" middle school

a socioeconomic system. A social outcome, such as the prevalence of an attribute in a population, is not simply the aggregation of individuals' attributes. Instead, each individual's behavior changes the conditions for other individuals' actions, adoption of certain attributes, and so on.

In order to investigate the role of the friendship paradox in such a context, I draw on a simple agent-based model of attribute adoption. Agent-based models simulate the emergence of broader macro-level phenomena from the bottom up. Agents, representing virtual counterparts of individual actors, are situated within social, structural, and normative environments (Gilbert 2008). The behavior of agents, in combination with interactions between agents, is shown to produce broader macro-level trends. This method is particularly appropriate for studying social dynamics.

Assuming that individuals adopt the attributes (or behaviors) of others, the question is whether the systematic exposure to others' attributes implied by the friendship paradox leads to a different distribution of the attributes in the population at large compared to a scenario where that distortion is absent.

As before, our simulation starts with the friendship network of "Cascade" middle school. By calibrating the structure of the simulation with an empirically observed network, I create realistic conditions for the aspect of the simulation where the friendship paradox applies, the degree distribution. I focus on the attribute $x$ of individuals and assume that $x$ can be changed $-x$ can stand for opinion, clothing style, or anything else - and that the values of $x$ are drawn from a normal distribution with mean score zero and a standard deviation of one. As before, the values of $x$ are allocated to individuals in the social network in such a way that $x$ is correlated with the number of friends (Degree) the individuals have. Once again, this is achieved with the built-in corgen command of the R-package ecodist. This time I artificially change the correlation between $x$ and Degree from 0 to 0.8 in steps of 0.2 .

Next, I apply a simple algorithm of attribute adoption in which at each point in time one randomly selected individual adopts the attribute of another individual. This can occur in two different ways: 1) A randomly selected actor copies the attribute of another randomly selected actor; or 2) A randomly selected actor copies the attribute of a randomly selected friend. In both cases, the individual who adopts the attribute and the individual whose attribute is adopted are selected at random, with the only difference being that in the latter scenario individuals can only copy from friends. In this way, the second scenario 
a) Degree

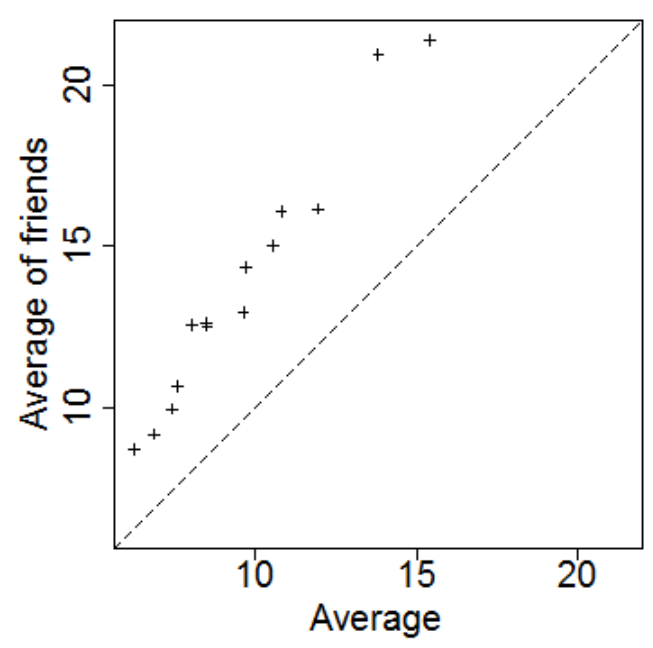

c) Closeness

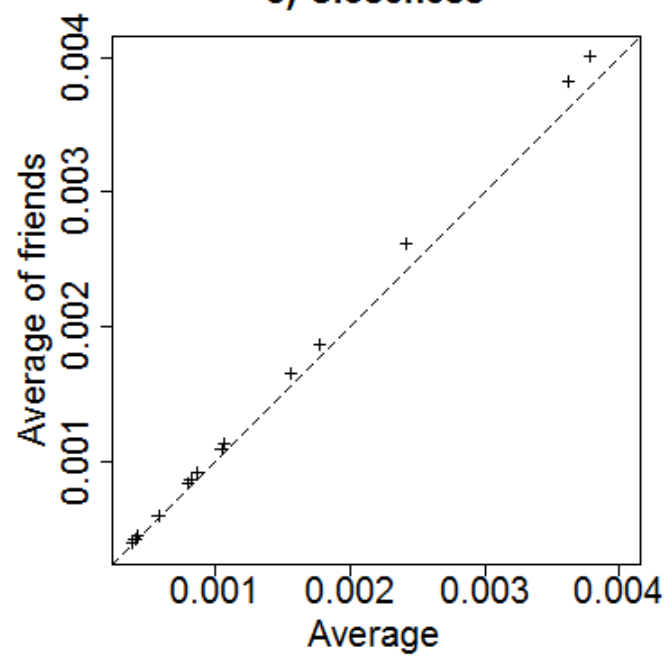

b) Betweenness

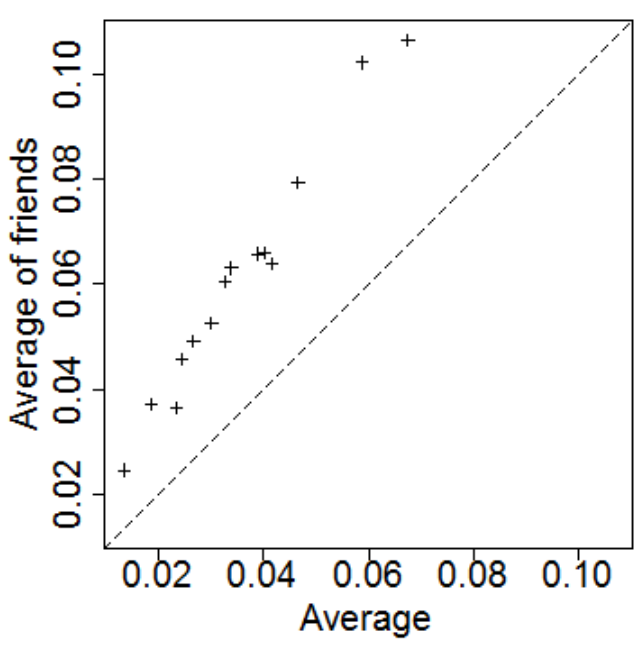

d) Eigenvector

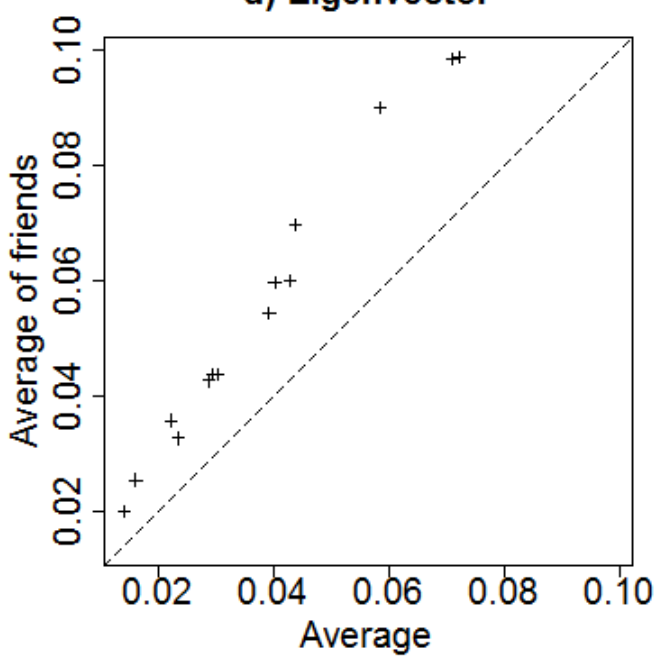

Figure 3: Average centrality and average centrality of friends in 14 schools in the United States 

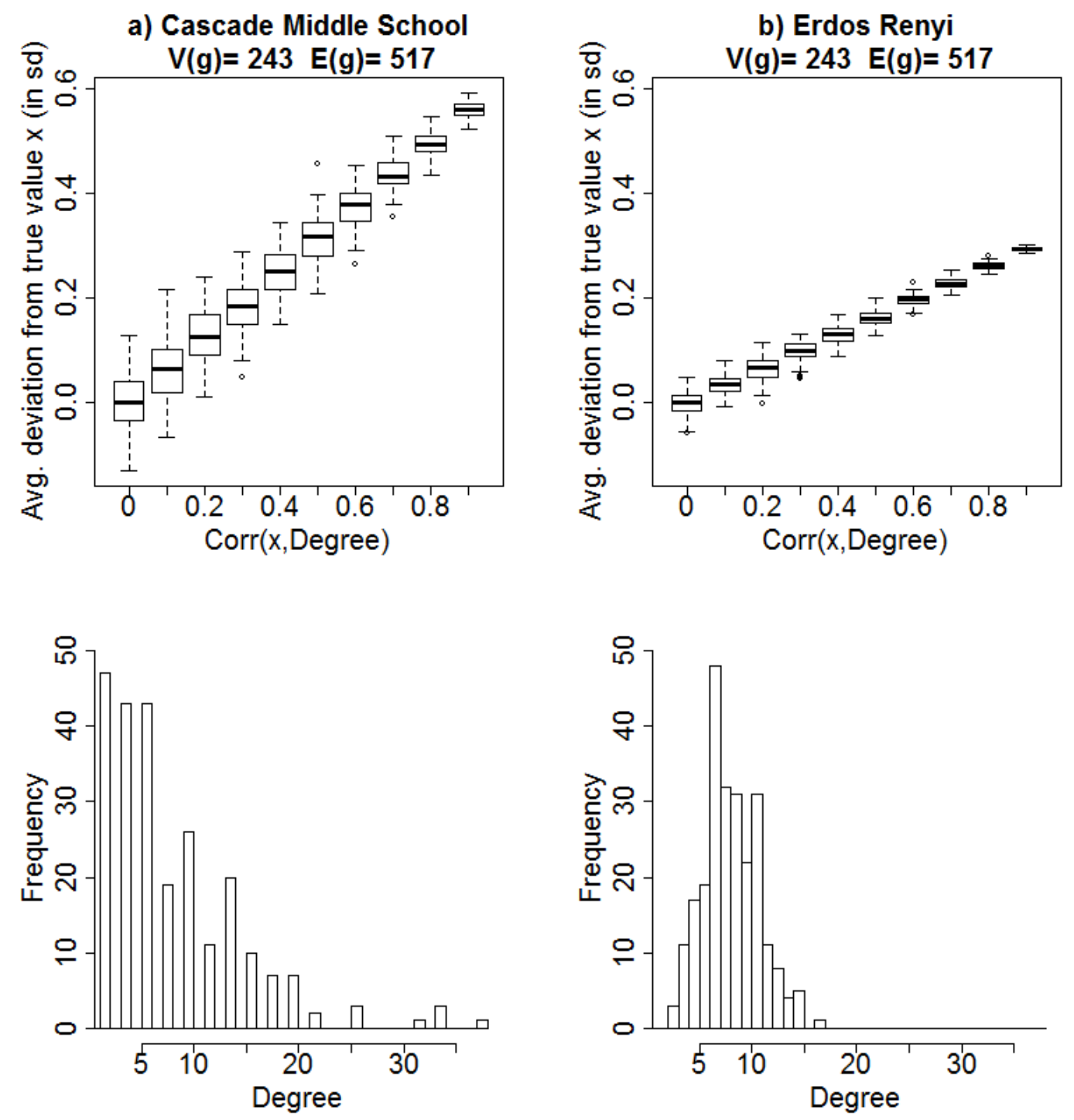

Figure 4: Experienced and true value of attribute $x$ (correlated with Degree) 
incorporates the friendship paradox, whereas the first scenario represents a baseline case where the friendship paradox is blended out. Comparing simulation results from both scenarios allows one to draw conclusions about the dynamic implications of the friendship paradox for attribute adoption.

Figure 5 shows results of a typical simulation run. One the left side I present the starting distribution of the attribute $x$. The shading on the right side of Figure 5 illustrates the results of a simulation run with no correlation between $x$ and Degree and the whole population as potential reference point (at each time one randomly selected actor copies the attribute of another randomly selected actor). For simplicity, there is no error in attribute adoption and no mutation. Neither would change the results, but their inclusion would distract from the point we want to make. The shading on in the right side of Figure 5 stands for the density distribution of attribute $x$. This is a simplified way to present the distribution of $x$, as on the left (start), but at different times during the simulation. All simulations are run in NetLogo 5.0.3 (Wilensky 1999) using the $R$ extension (Thiele, Kurth, and Grimm 2012) to facilitate seamless communication between the agent-based modeling and the statistics software.

Because actors only copy the attribute and make no alterations, the convergence in $x$ that is found makes sense. At some point in time, every actor has the same value on $x$. Although, in principle, all initial values of $x$ could be a point of convergence, the original distribution of $x$ matters a lot.

In Figure 6, I present repeated simulation results in which two parameters of the simulation are changed. First, as in the preceding analysis, I alter the correlation between $x$ and Degree. This is represented by a sub-graph for each correlation. Next, as described above, I restrict the reference group from which the randomly selected actor can copy the attribute to friends only. In the Random scenario (white bars), the selected actor copies the attribute from a randomly selected other actor. In the Friends scenario (grey bars), the selected actor copies the attribute from a randomly selected friend. For all combinations of correlation and scenario, the simulation is repeated 100 times with different starting values of $x$. A simulation run ends when convergence is reached and all actors have the same value on $x$. The boxplots in Figure 5 show the convergence values for the repeated simulations.

As one might expect, in the baseline Random scenario, the convergence values follow the same distribution of the initial values of $x$. Correlation between attribute score and network degree has no effect. When individuals copy the attribute value from a friend, the results are different. With increasing correlation $\operatorname{Corr}(x$, Degree $)$ at the start of the simulation, the convergence values of the simulation increase as well. Scores are typically above zero. This is interesting particularly because the original distribution of the attribute is exactly the same in both scenarios. This example shows that distortions introduced by the friendship paradox are not limited to the static perception of attributes or network positions. In fact, the distorted individual experience of the world that the paradox implies can actually change the world compared to the world that would have resulted from accurately perceptions.

\section{Discussion and conclusion}

A tradition in sociological thinking suggests that social phenomena (macro-level) both influence what individuals (micro-level) do and are the result of many individuals behaving in certain ways (Coleman 1990). Interdependencies between individuals are considered to be crucial to this process; the behavior of one individual changes the conditions for the actions of other individuals. In consequence, social phenomena are not simply the aggregate of separate individuals' decisions but the complex outcome of the dynamic interplay of individuals' behaviors. Agent-based models are a useful tool for analyzing such dynamics (Gilbert 2008).

This article focuses on an often neglected aspect of this phenomenon. Individuals base their decisions on what they think the social world looks like, but their assessments of others' attributes are systematically off from the true values in the population.

The friendship paradox is a purely structural perspective, postulating that in social network contexts, individuals - on average - experience their friends as having more friends than themselves. The deductive logic underlying this phe- 

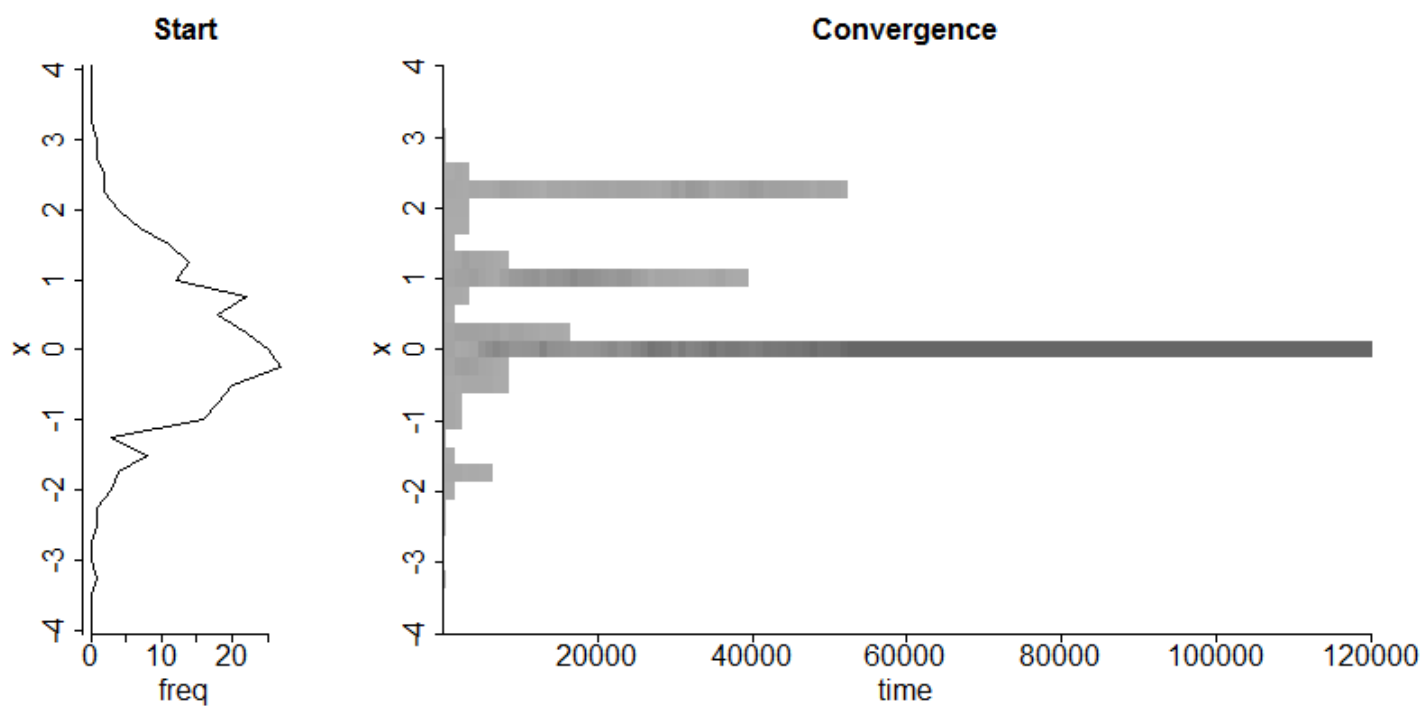

Figure 5: Starting distribution and convergence of a typical run
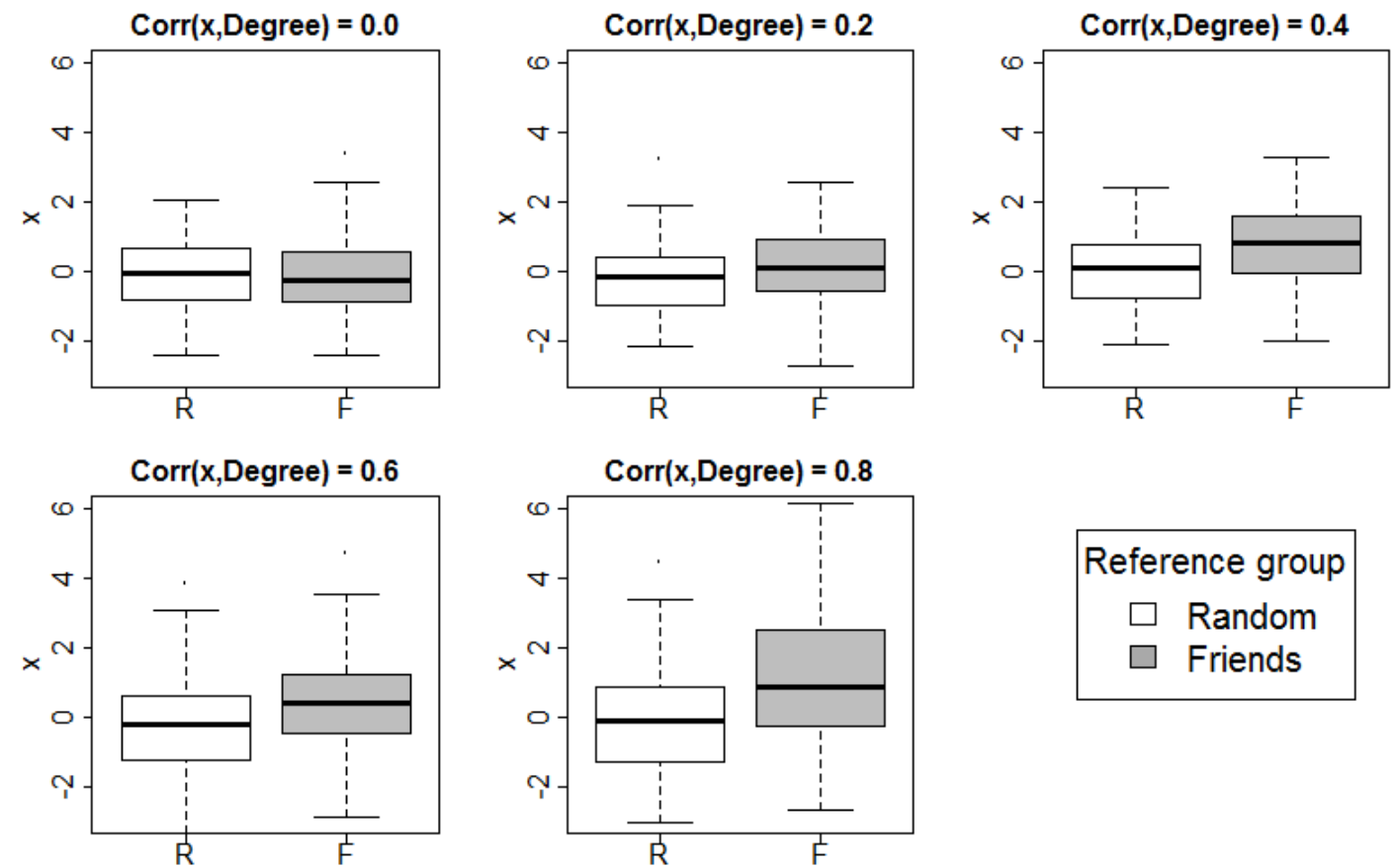

Figure 6: Convergence points with different correlations between attribute and degree 
nomenon is that heterogeneity in individuals numbers of friends translates into more popular individuals being present in more dyadic friendship relationships, with the result being a systematic mismatch in the distributions of number of friends and number of friends' friends.

So far, the friendship paradox had been exclusively thought of in terms of degree centrality. This article argues for a more general perspective and an extension of the concept to a wide range of network features and individual attributes. Simple embeddedness in a (heterogeneous) network structure generates conditions where individuals who are more important in terms of closeness, betweenness, and eigenvector centrality are more prevalent in friendship relationships as well. Hence the friends of a randomly selected sample of individuals are likely to occupy structural positions that are not representative of the population at large. On average, your friends not only have more friends (degree), but are also better informed (closeness), better intermediaries (betweenness), and more powerful (eigenvector) than you. Furthermore, the findings show that individuals' attributes are affected by the phenomenon. Even weak correlations between network position and actor attributes, which are present in many empirical settings, suffice to generate a situation where the friends of a randomly selected sample of individuals have significantly different attributes than the individuals in the original sample. Pure logic dictates that, for example, your friends are on average better looking than you.

Finally, I introduced an agent-based model of attribute adoption to illustrate the implications of the friendship paradox in a dynamic setting. The results clearly reveal that distortions of individuals' experience of the social world, introduced by nonrepresentative friends, persist and lead to different social outcomes compared to when individuals are not limited by their friendship relationships. Though an attribute may begin equally distributed among a virtual population, a simple process of attribute adoption leads to convergence at different values when the reference groups is changed. Hence, a situation where the friends of a randomly selected sample of individuals have significantly different attributes than individuals in the original sample, as implied by the friendship paradox, can change the trajectory of social processes in which individuals influence each other.

These findings have several important implications that have so far been mostly overlooked in the literature. For instance, individuals can be perfectly accurate in assessing themselves in relation to their friends but still be systematically off when it comes to the population at large, simply because their friends are not representative.

Several testable hypotheses follow from this. For example, nonrepresentative exposure to others' attributes will be most pronounced when both heterogeneity in degree distribution and correlation between attribute and degree are high. This can affect individuals' assessment of the real world. In contexts where individuals do not rely on friends for making assessments about the distribution of an attribute in a population, it may not matter that much. If, however, individuals heavily rely on friends to make inferences about a population as a whole, they will systematically overestimate the mean of such an attribute. In turn, the evaluation of one's own position or attribute in relation to the population as a whole will be misaligned. Hence, false assessments in relation to others should be more likely for attributes where individuals are less likely to obtain objective reference points from the population but have to draw on their experiences with their own friends instead.

In addition, the friendship paradox can be crucial for identifying hidden subpopulations. For example, in criminal contexts one often wants to find important criminals. A straightforward application of these insights would be to track down the co-offenders of a randomly selected group of offenders. By definition, the group of co-offenders will be more important in the criminal network as a whole and exhibit non-random attributes. The implications of the friendship paradox as discussed in this article offer tremendous potential for the identification of such actors in hidden populations. As I illustrate, the friends of a randomly selected group exhibit certain non-random attributes, which makes them nonrepresentative for the population at large. Sometimes, however, getting access to such nonrepresentative, important individuals is exactly what one wants. 


\section{References}

Bolland, John M. 1988. "Sorting out Centrality: An Analysis of the Performance of Four Centrality Models in Real and Simulated Networks." Social Networks 10(3): 233-253. http://dx.doi.org/10 1016/0378-8733(88)90014-7

Bonacich, Phillip. 1972. "Technique for Analyzing Overlapping Memberships." Sociological Methodology 4: 176-185. http://dx.doi org/10.2307/270732

. 1987. "Power and Centrality: A Family of Measures." American Journal of Sociology 92 (5): 1170-1182. http://dx.doi.org/10 $1086 / 228631$

Borgatti, Stephen P. and Martin G. Everett. 2006. "A Graph-Theoretic Perspective on Centrality." Social Networks 28 (4): 466-484. http://dx doi.org/10.1016/j.socnet.2005.11.005

Brass, Daniel, Joseph Galaskiewicz, Henrich Greve, and Wenpin Tsai. 2004. "Taking Stock of Networks and Organizations: A Multilevel Perspective." Academy of Management Journal 47 (6): 795-817. http: //dx.doi.org/10.2307/20159624

Christakis, Nicholas A. and James H. Fowler. 2010. "Social Network Sensors for Early Detection of Contagious Outbreaks." PLoS ONE 5 (9): e12948. http://dx.doi.org/10.1371/ journal.pone.0012948

Coleman, James. 1961. The Adolescent Society: The Social Life of the Teenager and Its Impact on Education. Glencoe, IL: Free Press of Glencoe.

- 1990. Foundations of Social Theory. Cambridge, MA: Harvard University Press.

Eom, Young-Ho and Hang-Hyun Jo. 2014. Generalized friendship paradox in complex networks. arXiv:1401.1458 [physics] Retrieved from http://arxiv.org/abs/1401.1458

Feld, Scott L. 1991. "Why Your Friends Have More Friends than You Do." American Journal of Sociology 96 (6): 1464-1477. http: //dx.doi.org/10.1086/229693

Feld, Scott L., and Bernard Grofman. 1977. "Variation in Class Size, the Class Size Paradox, and Some Consequences for Students." Research in Higher Education
6(3):215-22. http://dx.doi.org/10.1007/ BF00991287

Freeman, Linton C. 1978. "Centrality in Social Networks: Conceptual Clarification." Social Networks 1 (3): 215-239. http://dx.doi org/10.1016/0378-8733(78)90021-7

Friedkin, Noah. 1991. "Theoretical Foundations for Centrality Measures." American Journal of Sociology 96 (6): 1478-1504. http://dx doi.org/10.1086/229694

Gilbert, Nigel. 2008. Agent-Based Models. London: Sage.

Granovetter, Mark S. 1973. "The Strength of Weak Ties." American Journal of Sociology 78 (6): 1360-1380. http://dx.doi.org/10 $1086 / 225469$

Ibarra, Herminia. 1993. "Network Centrality, Power, and Innovation Involvement: Determinants of Technical and Administrative Roles." Academy of Management Journal 36 (3): 471501.

Marsden, Peter V. 2002. "Egocentric and Sociocentric Measures of Network Centrality." Social Networks 24 (4): 407-422. http: //dx.doi.org/10.2307/256589

Morselli, Carlo, Cynthia Giguère, and Katia Petit. 2007. "The Efficiency/security Trade-off in Criminal Networks." Social Networks 29 (1): 143-153. http://dx.doi.org/10.1016/ j.socnet.2006.05.001

Mulford, Matthew, John Orbell, Catherine Shatto, and Jean Stockard. 1998. "Physical Attractiveness, Opportunity, and Success in Everyday Exchange." American Journal of Sociology 103 (6): 1565-1592. http: //dx.doi.org/10.1086/231401

Newman, Mark E. J. 2003. "Ego-Centered Networks and the Ripple Effect." Social Networks 25(1): 83-95. http://dx.doi.org/10.1016/ S0378-8733(02) 00039-4

-. 2005. "A Measure of Betweenness Centrality Based on Random Walks." Social Networks 27 (1): 39-54. http://dx.doi.org/ $10.1016 /$ j.socnet. 2004.11.009

Noh, Jae Dong and Heiko Rieger. 2004. "Random Walks on Complex Networks." Physical Review Letters 92 (11): 118701. http://dx doi.org/10.1103/PhysRevLett.92.118701 
Rothenberg, Richard B., John J. Potterat, Donald E. Woodhouse, William W. Darrow, Stephen Q. Muth, and Alden S. Klovdahl. 1995. "Choosing a Centrality Measure: Epidemiologic Correlates in the Colorado Springs Study of Social Networks." Social Networks 17 (3-4): 273-297. http://dx.doi.org/10 1016/0378-8733(95)00267-R

Schelling, Thomas. 1971. "Dynamic Models of Segregation." Journal of Mathematical Sociology 1: 143-186. http://dx.doi.org/10 1080/0022250X.1971.9989794

Thiele, Jan C., Winfried Kurth, and Volker Grimm. 2012. "Agent-Based Modelling: Tools for Linking NetLogo and R." Journal of Artificial Societies and Social Simulation 15 (3): 8.

Tong, Stephanie Tom, Brandon Van Der Heide, Lindsey Langwell, and Joseph B. Walther. 2008. "Too Much of a Good Thing? The Relationship between Number of Friends and Interpersonal Impressions on Facebook." Journal of Computer-Mediated Communication 13 (3): 531-549. http://dx.doi.org/10.1111/ j.1083-6101.2008.00409.x

Tsai, Wenpin. 2001. "Knowledge Transfer in Intraorganizational Networks: Effects of Network Position and Absorptive Capacity on Business Unit Innovation and Performance." Academy of Management Journal 44 (5): 996-1004. http://dx.doi.org/10 $2307 / 3069443$

Valente, Thomas W., Kathryn Coronges, Cynthia Lakon, and Elizabeth Costenbader. 2008. "How Correlated Are Network Centrality Measures?" Connections 28 (1): 16-26.

Wilensky, Uri. 1999. "NetLogo." Evanston, IL: Northwestern University Center for Connected Learning and Computer-Based Modeling. http://ccl.northwestern.edu/ netlogo/.

Thomas U. Grund: Institute for Futures Studies, Stockholm, Sweden; Department of Sociology, Stockholm University, Sweden.

E-mail: thomas.u.grund@gmail.com. 